International Journal of Pure and Applied Mathematics

Volume 92 No. 3 2014, 359-367

ISSN: 1311-8080 (printed version); ISSN: 1314-3395 (on-line version)

url: http://www.ijpam.eu

doi: http://dx.doi.org/10.12732/ijpam.v92i3.4

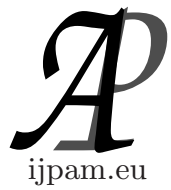

\title{
ON A SPECIAL CONCIRCULAR LIE-RECURRENCE IN A FINSLER SPACE
}

\author{
Vaishali Pandey ${ }^{1 \S}$, P.N. Pandey ${ }^{2}$ \\ ${ }^{1,2}$ Department of Mathematics \\ University of Allahabad \\ Allahabad, 211002, INDIA
}

\begin{abstract}
The concept of Lie-recurrence in a Finsler space was introduced by the second author [8] of the present paper in 1982. The Lie-recurrence in a Riemannian space was studied by K. L. Duggal [3] in 1992 but he used the term curvature inheriting symmetry in place of Lie-recurrence. K. L. Duggal also applied the theory to the study of fluid space time. Since then both the terms (Lie-recurrence and curvature inheriting symmetry) are in use. The present authors [11], Shivalika Saxena and P. N. Pandey [12], [13], C. K. Mishra and Gautam Lodhi [1] studied a Lie-recurrence (curvature inheriting symmetry) in a Finsler space and discussed the possibilities for contra and concurrent vector fields to generate Lie-recurrence. The present paper deals with a Lie-recurrence generated by a special concircular vector field and such Lie-recurrence is termed as a special concircular Lie-recurrence. We obtain certain results related to a special concircular Lie-recurrence in a general Finsler space as well as in birecurrent and bisymmetric Finsler spaces.
\end{abstract}

AMS Subject Classification: 53B40

Key Words: Finsler spaces, special concircular vector field, Lie-recurrence, curvature inheritance, birecurrent Finsler space, bisymmetric Finsler space

Received: October 8, 2013

(C) 2014 Academic Publications, Ltd. url: www.acadpubl.eu

${ }^{\S}$ Correspondence author 


\section{Introduction}

Let $F_{n}$ be an n-dimensional Finsler space equipped with a metric function $F\left(x^{i}, y^{i}\right)$ satisfying the requisite conditions[2], the corresponding metric tensor $g\left(x^{i}, y^{i}\right)$ and the Berwald connection $G\left(x^{i}, y^{i}\right)$. Henceforth all the geometric objects are assumed to be functions of line-element $\left(x^{i}, y^{i}\right)$, unless stated otherwise. Let the components of the metric tensor $g$ and coefficients of Berwald connection $G$ be denoted by $g_{i j}$ and $G_{j k}^{i}$ respectively. The metric function $F$ and the components $g_{i j}$ of the metric tensor are connected by

$$
\text { a) } g_{i j}=\frac{1}{2} \dot{\partial}_{i} \dot{\partial}_{j} F^{2}, \quad \text { b) } g_{i j} y^{i} y^{j}=F^{2}
$$

where $\dot{\partial}_{i} \equiv \frac{\partial}{\partial y^{1}}$.

The quantities $G_{j k h}^{i}$ defined by $G_{j k h}^{i}=\dot{\partial}_{h} G_{j k}^{i}$, are components of a symmetric tensor and satisfy

$$
G_{j k h}^{i} y^{h}=0,
$$

Berwald covariant derivative of a tensor field $T_{j}^{i}$ with respect to $x^{k}$ is defined by

$$
\mathrm{B}_{k} T_{j}^{i}=\partial_{k} T_{j}^{i}-\left(\dot{\partial}_{r} T_{j}^{i}\right) G_{k h}^{r} y^{h}+T_{j}^{r} G_{r k}^{i}-T_{r}^{i} G_{j k}^{r},
$$

where $\partial_{k} \equiv \frac{\partial}{\partial x^{k}}$.

The commutation formula for the operators $\dot{\partial}_{j}$ and $\mathbf{B}_{k}$ is given by

$$
\left(\dot{\partial}_{j} \mathrm{~B}_{k}-\mathrm{B}_{k} \dot{\partial}_{j}\right) X^{i}=G_{j k h}^{i} X^{h}
$$

while the Ricci-commutation formula is given by

$$
\left(\mathrm{B}_{j} \mathrm{~B}_{k}-\mathrm{B}_{k} \mathrm{~B}_{j}\right) X^{i}=H_{h k j}^{i} X^{h}-\left(\dot{\partial_{h}} X^{i}\right) H_{k j}^{h},
$$

$X^{i}$ being components of an arbitrary vector field. $H_{h k j}^{i}$ appearing in equation (5) are components of the Berwald curvature tensor. This tensor is skewsymmetric in last two lower indices and positively homogeneous of degree zero in $y^{i}$. This tensor and the tensor $H_{k j}^{i}$ appearing in equation (5) are connected by the following:

$$
\text { a) } H_{j k h}^{i} y^{j}=H_{k h}^{i}, \quad \text { b) } \dot{\partial}_{j} H_{k h}^{i}=H_{j k h}^{i} .
$$

The deviation tensor $H_{h}^{i}$ and the tensor $H_{k h}^{i}$ are related by

$$
\text { a) } H_{k h}^{i}=\frac{1}{3}\left(\dot{\partial_{k}} H_{h}^{i}-\dot{\partial}_{h} H_{k}^{i}\right), \quad \text { b) } H_{k h}^{i} y^{k}=H_{h}^{i} .
$$


Let a contravariant vector field $v^{i}\left(x^{j}\right)$ generates the infinitesimal transformation

$$
\bar{x}^{i}=x^{i}+\epsilon v^{i}\left(x^{j}\right),
$$

where $\epsilon$ is an infinitesimal constant. Let us denote the operator of Lie-differentiation with respect to this transformation by $£$. The Lie-derivative of an arbitrary tensor field $T_{j}^{i}$ with respect to above transformation [4] is given by

$$
£ T_{j}^{i}=v^{r} \mathrm{~B}_{r} T_{j}^{i}-T_{j}^{r} \mathrm{~B}_{r} v^{i}+T_{r}^{i} \mathrm{~B}_{j} v^{r}+\left(\dot{\partial}_{r} T_{j}^{i}\right) \mathrm{B}_{s} v^{r} y^{s},
$$

\section{Special Concircular Lie-Recurrence}

Let us consider a Finsler space admitting the infinitesimal transformation (8) generated by a special concircular vector field $v^{i}\left(x^{j}\right)$ characterized by

$$
\mathrm{B}_{k} v^{i}=\rho \delta_{k}^{i},
$$

where $\rho$ is not a constant.

Differentiating equation(10) partially with respect to $y^{j}$, we get

$$
\dot{\partial}_{j} \mathrm{~B}_{k} v^{i}=\left(\dot{\partial}_{j} \rho\right) \delta_{k}^{i},
$$

Using the commutation formula exhibited by equation (4), we have

$$
v^{r} G_{j k r}^{i}=\left(\dot{\partial}_{j} \rho\right) \delta_{k}^{i},
$$

Transvecting equation (12) by $y^{k}$ and using (2), we get $y^{i}\left(\dot{\partial}_{j} \rho\right)=0$, which implies $\dot{\partial}_{j} \rho=0$, This shows that $\rho$ is a function of positional co-ordinates $x^{i}$ only.The second author [7] proved that a special concircular vector field can not generate an affine motion in a Finsler space. This means $£ G_{j k}^{i} \neq 0$ for every special concircular transformation. Therfore, there is a possibility of existence of a special concircular transformation with respect to which $£ H_{j k h}^{i} \neq 0$.

Suppose the special concircular transformation (8) is a Lie-recurrence (curvature inheriting symmetry) in a Finsler space $F_{n}$, i.e.

$$
£ H_{j k h}^{i}=\Phi H_{j k h}^{i},
$$

where $\Phi$ is a non-zero scalar. The Second author [8] proved that the scalar function $\Phi$ is independent of $y^{i}$. In view of equation (9), equation (13) may be written as 
$v^{r} \mathrm{~B}_{r} H_{h j k}^{i}+\left(\dot{\partial}_{r} H_{h j k}^{i}\right) \mathrm{B}_{s} v^{r} y^{s}-H_{h j k}^{r} \mathrm{~B}_{r} v^{i}+H_{r j k}^{i} \mathrm{~B}_{h} v^{r}+H_{h r k}^{i} \mathrm{~B}_{j} v^{r}+H_{h j r}^{i} \mathrm{~B}_{k} v^{r}=$ $\Phi H_{h j k}^{i}$,

Using equation (10) and the fact that the curvature tensor $H_{h j k}^{i}$ is positively homogeneous of degree zero in $y^{i}$, we get

$$
v^{r} \mathrm{~B}_{r} H_{h j k}^{i}=(\phi-2 \rho) H_{h j k}^{i} .
$$

Differentiating equation (10) covariantly with respect to $x^{j}$, we get

$$
\mathrm{B}_{j} \mathrm{~B}_{k} v^{i}=\rho_{j} \delta_{k}^{i}
$$

where $\rho_{j}=\mathrm{B}_{j} \rho$.

Taking skew- symmetric part of equation (15), we have

$$
\mathrm{B}_{j} \mathrm{~B}_{k} v^{i}-\mathrm{B}_{k} \mathrm{~B}_{j} v^{i}=\rho_{j} \delta_{k}^{i}-\rho_{k} \delta_{j}^{i} .
$$

Using the commutation formula exhibited by equation (5), we get

$$
v^{r} H_{r k j}^{i}=\rho_{j} \delta_{k}^{i}-\rho_{k} \delta_{j}^{i}
$$

Contracting the indices $i$ and $j$ in equation (16), we have

$$
v^{r} H_{r k}=-(n-1) \rho_{k},
$$

where $H_{r k}$ is the Ricci tensor defined by $H_{r k}=H_{r k s}^{s}$.

Using equation (17) in equation (16), we find

$$
\left((n-1) H_{r k j}^{i}+H_{r j} \delta_{k}^{i}-H_{r k} \delta_{j}^{i}\right) v^{r}=0 .
$$

Now,

$$
£ \rho_{k}=v^{m} \mathbf{B}_{m} \rho_{k}+\left(\partial_{r} \rho_{k}\right) \mathbf{B}_{s} v^{r} y^{s}+\rho_{r} \mathbf{B}{ }_{k} v^{r},
$$

Using equation (10) and the fact that $\rho_{k}$ is homogenous of degree zero in $y^{i}$, we get

$$
£ \rho_{k}=v^{m} \mathbf{B}_{m} \rho_{k}+\rho \rho_{k} .
$$

Transvecting equation (14) by $v^{h}$, we have

$$
v^{h} v^{r} \mathbf{B}_{r} H_{h j k}^{i}=(\phi-2 \rho) H_{h j k}^{i} v^{h} .
$$

Differentiating equation (16) covariantly with respect to $x^{m}$, we have

$$
\mathbf{B}_{m} v^{r} H_{r k j}^{i}+v^{r} \mathbf{B}_{m} H_{r k j}^{i}=\mathbf{B}_{m} \rho_{j} \delta_{k}^{i}-\mathbf{B}_{m} \rho_{k} \delta_{j}^{i},
$$


Transvecting by $v^{m}$ and using equation (10), we have

$$
v^{r} \rho H_{r k j}^{i}+v^{m} v^{r} \mathbf{B}_{m} H_{r k j}^{i}=v^{m}\left(\mathbf{B}_{m} \rho_{j} \delta_{k}^{i}-\mathbf{B}_{m} \rho_{k} \delta_{j}^{i}\right),
$$

In view of equation (20), equation (21) implies

$$
v^{r} \rho H_{r k j}^{i}+(\phi-2 \rho) H_{r k j}^{i} v^{r}=v^{m} \mathbf{B}_{m}\left(\rho_{j} \delta_{k}^{i}-\rho_{k} \delta_{j}^{i}\right),
$$

Using equation (16) in equation (22), we have

$$
\rho\left(\rho_{j} \delta_{k}^{i}-\rho_{k} \delta_{j}^{i}\right)+(\phi-2 \rho)\left(\rho_{j} \delta_{k}^{i}-\rho_{k} \delta_{j}^{i}\right)=v^{m} \mathbf{B}_{m}\left(\rho_{j} \delta_{k}^{i}-\rho_{k} \delta_{j}^{i}\right),
$$

Contracting the indices $i$ and $j$ in equation (23), we get

$$
v^{m} \mathbf{B}_{m} \rho_{k}=(\phi-\rho) \rho_{k},
$$

Using equation (24) in equation (19), we have

$$
£ \rho_{k}=\phi \rho_{k} .
$$

This leads to:

Theorem 1. If a Finsler space admits a special concircular Lie-recurrence characterized by equation (10) and equation (13), the covariant derivative of scalar $\rho$ appearing in equation (10) is Lie-recurrent with respect to the Lierecurrence.

\section{Special Concircular Lie-Recurrence in a Birecurrent Finsler Space}

Let us consider a birecurrent Finsler space $F_{n}$ characterized [7] by

$$
\mathrm{B}_{l} \mathrm{~B}_{m} H_{j k h}^{i}=a_{l m} H_{j k h}^{i},
$$

where $a_{l m}$ are components of a non-zero covariant tensor of type $(0,2)$ and $H_{j k h}^{i} \neq 0$.

suppose that this space admits a special concircular Lie-recurrence. Then we have equation (14).

Differentiating equation (14) covariantly with respect to $x^{m}$, we get

$$
\mathbf{B}_{m} v^{r} \mathbf{B}_{r} H_{h j k}^{i}+v^{r} \mathbf{B}_{m} \mathbf{B}_{r} H_{h j k}^{i}=\left(\phi_{m}-2 \rho_{m}\right) H_{h j k}^{i}+(\phi-2 \rho) \mathbf{B}_{m} H_{h j k}^{i},
$$


where $\phi_{m}=\mathrm{B}_{m} \phi$.

Using equation (10) and equation (26) in equation (27), we have

$$
\left(v^{r} a_{m r}-\phi_{m}+2 \rho_{m}\right) H_{h j k}^{i}=(\phi-3 \rho) \mathrm{B}_{m} H_{h j k}^{i} .
$$

A birecurrent Finsler space is non-flat i.e. $H_{j k h}^{i} \neq 0$. Also, it is not symmetric i.e. $\mathbf{B}_{m} H_{h j k}^{i} \neq 0$, for $\mathbf{B}_{m} H_{h j k}^{i}=0$ implies $\mathbf{B}_{l} \mathbf{B}_{m} H_{h j k}^{i}=0$, in view of (26), gives $a_{l m}=0$, a contradiction.

Therefore, equation (28) implies either of the following conditions

(i) $\phi-3 \rho=0, \quad v^{r} a_{m r}-\phi_{m}+2 \rho_{m}=0$,

(ii) $\phi-3 \rho \neq 0, \quad v^{r} a_{m r}-\phi_{m}+2 \rho_{m} \neq 0$.

we can write the condition $(i)$ as $\phi=3 \rho, v^{r} a_{m r}=\rho_{m}$.

Let us consider the condition $(\mathrm{ii})$. In this case, equation (28) may be written as

$$
\mathbf{B}_{m} H_{h j k}^{i}=\frac{\left(v^{r} a_{m r}-\phi_{m}+2 \rho_{m}\right)}{\phi-3 \rho} H_{h j k}^{i},
$$

which shows that the space is recurrent, The second author [6] proved that a recurrent Finsler space does not admit a special concircular vector field, which implies that a recurrent Finsler space does not admit a special concircular Lierecurrence. Therefore, the pair of conditions $(i i)$ is not possible. Hence, we may conclude:

Theorem 2. A birecurrent Finsler space $F_{n}$ admitting a special concircular Lie-recurrence necessarily satisfies the conditions $\phi=3 \rho$ and $v^{r} a_{m r}=\rho_{m}$.

Taking skew-symmetric part of equation (26) and using the Ricci-commutation formula exhibited by (5), we have

$$
H_{j k h}^{r} H_{r m l}^{i}-H_{r k h}^{i} H_{j m l}^{r}-H_{j r h}^{i} H_{k m l}^{r}-H_{j k r}^{i} H_{h m l}^{r}-\left(\dot{\partial}_{r} H_{j k h}^{i}\right) H_{m l}^{r}=A_{l m} H_{j k h}^{i},
$$

where $A_{l m}=a_{l m}-a_{m l}$. Operating both sides of equation (30) by the operator $£$ and using equation (13), we get

$$
£ A_{l m}=\phi A_{l m}
$$

This leads to:

Theorem 3. The skew-symmetric part of the recurrence tensor $a_{l m}$ of a birecurrent Finsler space admitting a special concircular Lie-recurrence is Lie-recurrent with respect to the Lie-recurrence. 


\section{Special Concircular Lie-Recurrence in a Bisymmetric Finsler Space}

Let us consider a bisymmetric Finsler space $F_{n}$ characterized by

$$
\mathrm{B}_{m} \mathbf{B}_{l} H_{h j k}^{i}=0 .
$$

Differentiating equation (14), covariantly with respect to $x^{m}$, we have

$$
\mathbf{B}_{m} v^{r} \mathbf{B}_{r} H_{h j k}^{i}+v^{r} \mathbf{B}_{m} \mathbf{B}_{r} H_{h j k}^{i}=\left(\phi_{m}-2 \rho_{m}\right) H_{h j k}^{i}+(\phi-2 \rho) \mathbf{B}_{m} H_{h j k}^{i}
$$

Using equations (10) and (31) in (32),we get

$$
(\phi-3 \rho) \mathbf{B}_{m} H_{h j k}^{i}=\left(2 \rho_{m}-\phi_{m}\right) H_{h j k}^{i}
$$

If $\phi=3 \rho$, equation (33) reduces to $\rho_{m} H_{h j k}^{i}=0$, which implies $H_{h j k}^{i}=0$ for $\rho_{m} \neq 0$. Thus we conclude:

Theorem 4. A bisymmetric Finsler space $F_{n}$ admitting a special concircular Lie-recurrence with condition $\phi=3 \rho$ is flat.

If $\phi=2 \rho$ then $\phi_{m}-2 \rho_{m}=0$. Therefore equation (33) may be written as

$$
\mathrm{B}_{m} H_{h j k}^{i}=0 \text {. }
$$

This shows that the space is symmetric. Thus, we see that a bisymmetric Finsler space admitting a special concircular Lie-recurrence with $\phi=2 \rho$ is a symmetric space admitting a special concircular Lie-recurrence.

In view of a result due to the second author [10], a symmetric Finsler space $F_{n}(n>2)$ admitting a special concircular transformation is a Riemannian space of constant Riemannian curvature.

Therefore, we may conclude:

Theorem 5. A bisymmetric Finsler space $F_{n}(n>2)$ admitting a special concircular Lie-recurrence with $\phi=2 \rho$ is a Riemannian space with constant Riemannian curvature.

If $\phi \neq 2 \rho$ and $\phi \neq 3 \rho$, then equation (33) may be written as

$$
\mathbf{B}_{m} H_{h j k}^{i}=\frac{\left(2 \rho_{m}-\phi_{m}\right)}{\phi-3 \rho} H_{h j k}^{i} .
$$

This shows that the space is recurrent, but a recurrent space admitting a special concircular Lie-recurrence does not exist.

Hence we may conclude: 
Theorem 6. A bisymmetric Finsler space $F_{n}(n>2)$ can not admit a special concircular Lie-recurrence if $\phi$ is neither $2 \rho$ nor $3 \rho$.

From Theorems 4,5 and 6, we may conclude:

Theorem 7. A bisymmetric Finsler space $F_{n}(n>2)$ admitting a special concircular Lie-recurrence is either flat or a Riemannian space of constant Riemannian curvature.

\section{References}

[1] C.K.Mishra, Gautam Lodhi, On Curvature Inheriting Symmetry in Finsler space, Acta Universitatis Apulensis, 30 (2012), 39-48.

[2] H. Rund, The differential geometry of Finsler spaces, Springer-Verlag, Berlin (1959).

[3] K.L.Duggal, Curvature inheriting symmetry in Riemannian space with application of fluid space time, J. Math. Phys., 33(9) (1992),2989-2996.

[4] K. Yano, The Theory of Lie derivatives and its applications, North-Holland Publishing co. Amsterdam (1957).

[5] P. N. Pandey, On bisymmetric Finsler manifold, Math education, 11 (4) (1977),77-80.

[6] P. N. Pandey, A recurrent Finsler manifold with a concircular vector field, Acta. Math. Acad. Sci. (Hungar.), 35 (3-4) (1980), 465-466.

[7] P. N. Pandey, Certain types of affine motion in a Finsler manifold. I, Colloq. math., 49 (1985),243-252.

[8] P. N. Pandey, On Lie-recurrent Finsler manifold, Indian Journal of Mathematics., 24 (1982), 135-143.

[9] P. N. Pandey, On some Finsler spaces of scalar curvature, Prog. of Maths., 18 (1) (1984), 41-48.

[10] P. N. Pandey, A symmetric Finsler manifold with a concircular vector field, Proc. Nat. Acad. Sci. (India), 54 (1984), 271-273.

[11] P. N. Pandey, Vaishali Pandey, On $\widetilde{K}$-curvature inheritance in a Finsler space, Int. Acad. Phy. Sci.13(4) (2009), 395-400. 
[12] P. N. Pandey, S. Saxena, On Projective N-curvature inheritance, Acta. Math. Hungar., 136(1-2) (2012), 30-38.

[13] Shivalika Saxena, P. N. Pandey, On Lie-recurrence in Finsler spaces, Diff. Geo. Dyn. Sys., 13 (2011), 201-207. 
